\title{
New study of the line profiles of sodium perturbed by $\mathrm{H}_{2}^{\star}$
}

\author{
N. F. Allard ${ }^{1,2}$, F. Spiegelman ${ }^{3}$, T. Leininger ${ }^{3}$, and P. Molliere ${ }^{4}$ \\ 1 GEPI, Observatoire de Paris, PSL Research University, UMR 8111, CNRS, Sorbonne Paris Cité, 61, \\ Avenue de l'Observatoire, 75014 Paris, France \\ e-mail: nicole.allard@obspm. fr \\ 2 Institut d'Astrophysique de Paris, UMR7095, CNRS, Université Paris VI, 98bis Boulevard Arago, Paris, France \\ ${ }^{3}$ Laboratoire de Physique et de Chimie Quantique, Université de Toulouse (UPS) and CNRS, 118 route de Narbonne, \\ 31400 Toulouse, France \\ ${ }^{4}$ Leiden Observatory, Leiden University, Postbus 9513, 2300, RA Leiden, The Netherlands
}

Received 1 April 2019 / Accepted 2 July 2019

\begin{abstract}
The opacity of alkali atoms, most importantly of $\mathrm{Na}$ and $\mathrm{K}$, plays a crucial role in the atmospheres of brown dwarfs and exoplanets. We present a comprehensive study of $\mathrm{Na}-\mathrm{H}_{2}$ collisional profiles at temperatures from 500 to $3000 \mathrm{~K}$, the temperatures prevailing in the atmosphere of brown dwarfs and Jupiter-mass planets. The relevant $\mathrm{H}_{2}$ perturber densities reach several $10^{19} \mathrm{~cm}^{-3}$ in hot $\left(T_{\text {eff }} \gtrsim 1500 \mathrm{~K}\right)$ Jupiter-mass planets and can exceed $10^{20} \mathrm{~cm}^{-3}$ for more massive or cooler objects. Accurate pressure-broadened profiles that are valid at high densities of $\mathrm{H}_{2}$ should be incorporated into spectral models. Unified profiles of sodium perturbed by

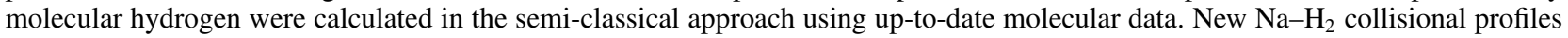
and their effects on the synthetic spectra of brown dwarfs and hot Jupiters computed with petitCODE are presented.
\end{abstract}

Key words. line: profiles - molecular data - brown dwarfs

\section{Introduction}

Alkali atoms are an important class of absorbers for modeling and understanding the spectra of self-luminous objects such as brown dwarfs and directly imaged planets. The wings of the sodium and potassium resonance lines in the optical are particularly important because they serve as a source of pseudo-continuum opacity, reaching into the near-infrared (NIR) wavelengths in the case of potassium. The relevance of these alkali species for the atmospheres of such self-luminous objects is studied and discussed in detail in Burrows et al. (2001). Moreover, it has been shown that the exact shape of the wings of the alkali lines, especially the red wings of the K doublet, affects the atmospheric structure, and that different treatments of the line wings can lead to differences in the temperature profiles at larger pressures (see, e.g., Baudino et al. 2017).

The class of transiting exoplanets (especially the so-called hot Jupiters) is also affected by the presence of the alkalis. Key observations with space- and ground-based telescopes have shed light on the conditions and composition of their atmospheres. Sodium was first detected in the atmosphere of HD209458b (Charbonneau et al. 2002), and is now routinely detected in many hot Jupiters from the ground and from space at high and low resolution. For examples, see Snellen et al. (2008), and the compilation of spectra in Sing et al. (2016) and Pino et al. (2018). Recently, the wing absorption of sodium was probed from the ground with the FORS2 observations by Nikolov et al. (2018).

For irradiated gas planets of intermediate temperature, the absorption of stellar light by the $\mathrm{Na}$ and $\mathrm{K}$ doublet line wings

\footnotetext{
* Opacity tables are only available at the CDS via anonymous ftp to cdsarc.u-strasbg. fr (130.79.128.5) or via http://cdsarc. u-strasbg.fr/viz-bin/qcat?J/A+A/628/A120
}

in the optical represents an important heating source (e.g., Mollière et al. 2015). Moreover, in the absence of strong cloud absorption, $\mathrm{Na}$ and $\mathrm{K}$ are the only significant absorbers of the flux of the host star in the optical. For higher planetary temperatures $(T \gtrsim 2000 \mathrm{~K})$, additional absorption by metal oxides, hydrides, atoms, and ions can become important (see, e.g., Arcangeli et al. 2018; Lothringer et al. 2018 and Lothringer \& Barman 2019). Coupling of $\mathrm{Na}$ and $\mathrm{K}$ abundance to energy transfer causes the atmospheric temperature structures of hot Jupiters to be very sensitive to the shapes of the $\mathrm{Na}$ and $\mathrm{K}$ doublet lines. For special chemical conditions where the planetary cooling opacity is low, the heating by alkali atoms can even create inversions (Mollière et al. 2015). For self-luminous planets, the alkali opacities are important by virtue of their line wings as well. The red wings of the $\mathrm{K}$ doublet line in particular can control the flux escaping the planets in the $Y$ band, which can also affect the planetary structure (Baudino et al. 2017).

In continuation with Allard et al. (2016) we present new unified line profiles of neutral $\mathrm{Na}$ perturbed by $\mathrm{H}_{2}$ using ab initio $\mathrm{Na}-\mathrm{H}_{2}$ potentials and transition dipole moments. In Allard et al. (2003) we presented absorption profiles of sodium perturbed by molecular hydrogen. The line profiles were calculated in a unified line shape semi-classical theory (Allard et al. 1999) using Rossi \& Pascale (1985) pseudo-potentials. Reliable calculations of pressure broadening in the far spectral wings require accurate potential-energy curves describing the interaction of the ground and excited states of $\mathrm{Na}$ with $\mathrm{H}_{2}$. In Allard et al. (2003) and Tinetti et al. (2007) we presented the first applications of semiclassical profiles of sodium and potassium perturbed by molecular hydrogen to the modeling of brown dwarfs and extra-solar planets. The $3 \mathrm{~s}-3 \mathrm{p}$ resonance line profiles were calculated using the Rossi \& Pascale (1985) pseudo-potentials (hereafter labeled RP85). Ab initio calculations of the potentials (hereafter 
labeled FS11) of $\mathrm{Na}-\mathrm{H}_{2}$ were computed by one of us (FS) and compared to pseudo-potentials of Rossi \& Pascale (1985) in Allard et al. (2012a). We highlighted the regions of interest near the $\mathrm{Na}-\mathrm{H}_{2}$ quasi-molecular satellites for comparison with previous results described in Allard et al. (2003). We also compared with laboratory absorption spectra. We extended this work to other excited states to allow a comprehensive determination of the spectrum (Allard et al. 2012b). Nevertheless, tables of $\mathrm{Na}-\mathrm{H}_{2}$ absorption coefficients which are currently used for the construction of model atmospheres and synthetic spectra which have been generated from the line profiles reported in Allard et al. (2003) needed to be up to date.

We have now extended the calculations of the $\mathrm{Na}-\mathrm{H}_{2}$ potential energy surfaces to the $5 \mathrm{~s}$ state, and improved the accuracy for lower states. The new ab initio calculations of the potentials are carried out for the $C_{2 v}$ (T-shape) symmetry group and the $C_{\infty v}$ (linear) symmetry group. In this paper we restrict our study to the resonance $3 \mathrm{~s}-3 \mathrm{p}$ line and will use the potentials of the more excited states described in Sect. 2 for a subsequent paper devoted to the line profiles of the sodium lines in the NIR. In addition, the transition dipole moments for the resonance line absorption, as a function of the geometry of the $\mathrm{Na}-\mathrm{H}_{2}$ system are presented. The improvement over our previous work (Allard et al. 2012a) consists in a better determination of the long-range part of the $\mathrm{Na}-\mathrm{H}_{2}$ potential curves. The inclusion of the spin-orbit coupling together with this improvement allow the determination of the individual line widths of the two components of the resonance $3 \mathrm{~s}-3 \mathrm{p}$ doublet (Sect. 3). We illustrate the evolution of the absorption spectra of $\mathrm{Na}-\mathrm{H}_{2}$ collisional profiles for the densities and temperatures prevailing in the atmospheres of cool brown dwarf stars and extrasolar planets. The new opacity tables of $\mathrm{Na}-\mathrm{H}_{2}$ have been incorporated into atmosphere calculations of self-luminous planets and hot Jupiters. The atmospheric models presented in Sect. 4 have been calculated with petitCODE, a well-tested code that solves for the 1D structures of exoplanet atmospheres in radiative-convective and thermochemical equilibrium. Gas and optionally cloud opacities can be included, and scattering is treated in the structure and spectral calculations. petitCODE is described in Mollière et al. $(2015,2017)$.

\section{2. $\mathrm{Na}-\mathrm{H}_{2}$ potentials including spin-orbit coupling}

The ab initio calculations of the potentials (hereafter labeled S17) were carried out for the $C_{2 v}$ (T-shape) symmetry group and the $C_{\infty v}$ (linear) symmetry group in a wide range of distances $R$ between the $\mathrm{Na}$ atom and the center-of-mass of the $\mathrm{H}_{2}$ molecule. The potentials were calculated with the MOLPRO package (Werner et al. 2012) and are shown in Figs. 1-3. In the calculation of the complex, the bond length of $\mathrm{H}_{2}$ was kept fixed at the equilibrium value $r_{\mathrm{e}}=1.401 \mathrm{a} . \mathrm{u}$. and the approach is along the $z$ coordinate axis. As in our previous calculations on $\mathrm{KH}_{2}$ (Allard et al. 2007a) and $\mathrm{NaH}_{2}$ (Allard et al. 2012a), we used a single active electron description of the sodium atom complemented with a core polarisation operator to include the core response. The effective core potential is the ECP10SDF effective core potential of the Stuttgart group (Nicklass et al. 1995). The core-polarization uses the formulation of Müller et al. (1984) with the parameters $\alpha=0.997 a_{0}^{3}$ and $\rho_{\mathrm{c}}=0.62$ for the core polarizability and the cut-off parameter of the CPP operator, respectively (corresponding to the smooth cut-off expression defined in MOLPRO). We used relatively extensive Gaussiantype basis sets (GTOs) to describe the three active electrons, namely $8 \mathrm{~s} 5 \mathrm{p} 6 \mathrm{~d} 8 \mathrm{f} 4 \mathrm{~g}$ for $\mathrm{Na}$ and an spdf AV5Z basis set for each hydrogen atom. With this basis set, the one-electron scheme for
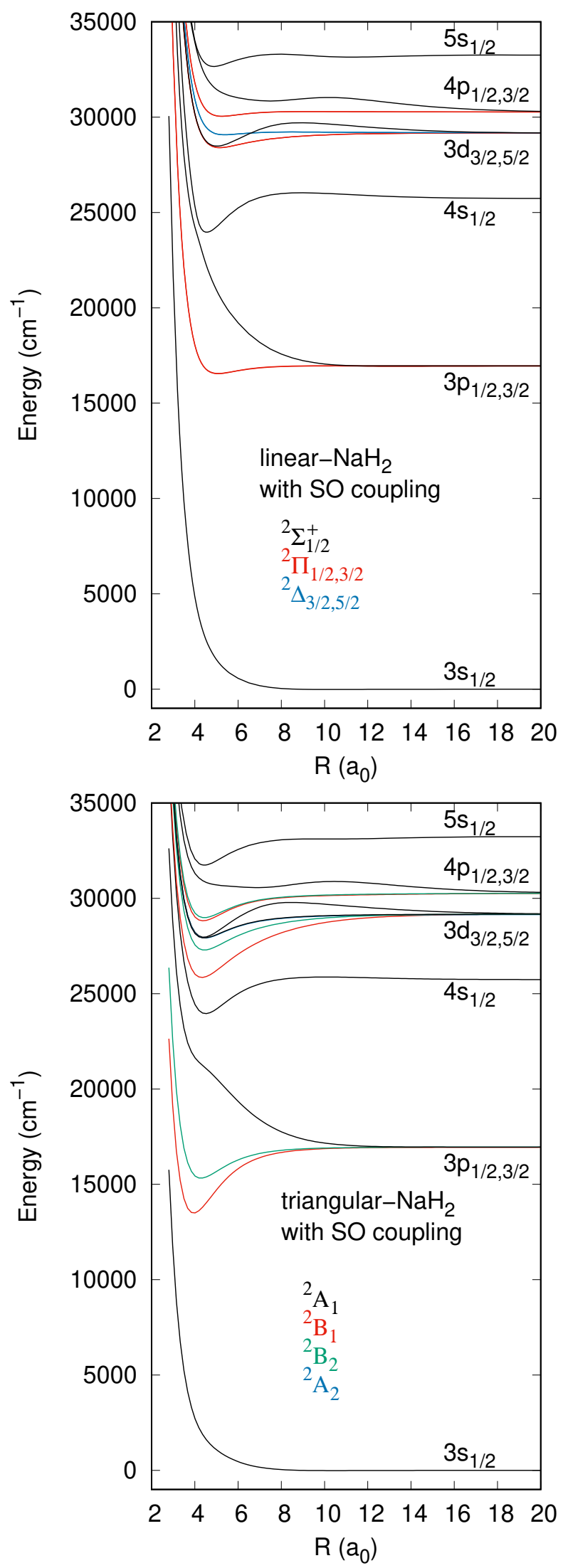

Fig. 1. Potential curves of the $\mathrm{Na}-\mathrm{H}_{2}$ molecule for the $C_{\infty v}$ (top) and $C_{2 v}$ symmetries (bottom). For the $C_{2 v}$ case, the symmetry labeling corresponds to the convention of the reference plane as that containing the molecule and may be different from that of previous publications. We note that states $1^{2} \mathrm{~A}_{2}$ and $4^{2} \mathrm{~A}_{1}$ correlated with the $3 \mathrm{~d}$ asymptote are superimposed at the scale of the figure. 
N. F. Allard et al.: New study of the line profiles of sodium perturbed by $\mathrm{H}_{2}$

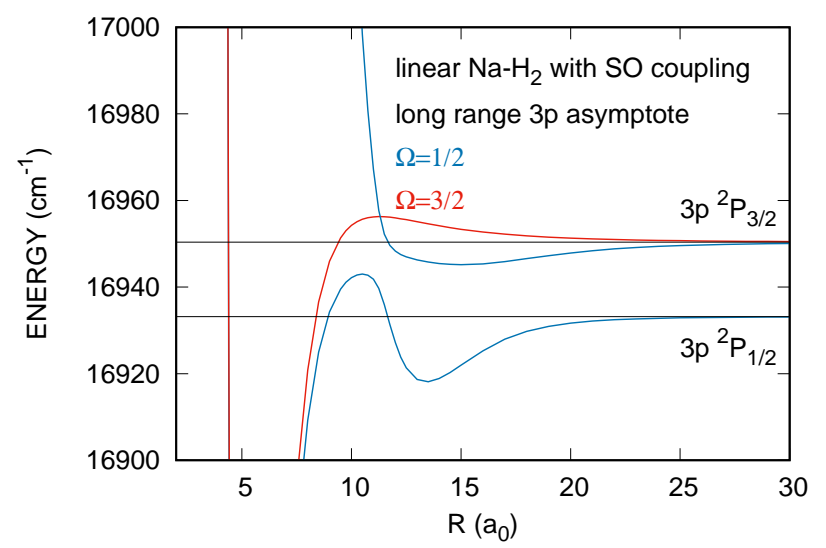

Fig. 2. Long-range potential curves of $\mathrm{NaH}_{2}$ correlated with the $3 \mathrm{p}_{1 / 2}$ and $3 \mathrm{p}_{3 / 2}$ asymptotes in $C_{\infty v}$ symmetry.

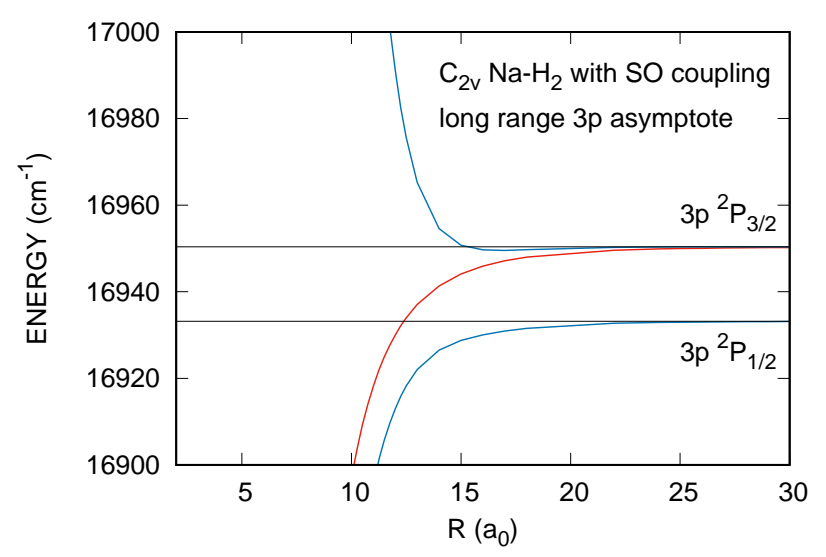

Fig. 3. Long-range potential curves of $\mathrm{NaH}_{2}$ correlated with the $3 \mathrm{p}_{1 / 2}$ and $3 \mathrm{p}_{3 / 2}$ asymptotes in $C_{2 v}$ symmetry.

sodium describes the transitions to the excited states of $\mathrm{Na}$ with an accuracy better than $25 \mathrm{~cm}^{-1}$ up to $5 \mathrm{~s}$, as illustrated in Table 1 and provides a fair account of correlation for the electrons of $\mathrm{H}_{2}$.

The determination of the electronic structure of $\mathrm{NaH}_{2}$ was carried out using the Multi Reference Configuration Interaction (MRCI) scheme of the MOLPRO package using the orbitals of $\mathrm{NaH}_{2}^{+}$, which should provide an adequate Molecular Orbitals (MOs) description of the excited orbitals of the neutral molecule. For $C_{\infty v}$ and $C_{2 v}$, the same symmetry subgroup is used in MOLPRO, namely $C_{2 v}$, the irreducible representations (irrep) of which correspond to $\Sigma$ (and one $\Delta$ ), $\Pi_{x}, \Pi_{\mathrm{y}}$, and $\Delta$ states, respectively, for the linear case, and $A_{1}, B_{2}, B_{1}$, and $A_{2}$ states, respectively, for the isosceles case. The MRCI was generated from a Complete Active Space (CAS) involving three active electrons in $12,8,8$, and 4 orbitals in each of the four irreps, respectively. This means that the generating CAS involves the valence orbitals of $\mathrm{H}_{2}$ as well as the $3 \mathrm{~s}, 3 \mathrm{p}, 4 \mathrm{~s}, 3 \mathrm{~d}, 4 \mathrm{p}, 5 \mathrm{~s}$ orbitals of sodium and even beyond. The MRCI space contains all simple and double excitations with respect to the CAS space (namely around $5 \times 10^{5}$ configurations for each irrep).

Since we want to address spectral regions close to the line center of the atomic absorbing lines $3 p$ and $4 p$, we incorporated SO coupling within a variant of the atom-in-molecule-like scheme introduced by Cohen \& Schneider (1974). This scheme relies on a monoelectronic formulation of the spin-orbit coupling operator

$H_{\mathrm{SO}}=\sum_{i} h_{\mathrm{SO}}(i)=\sum_{i} \zeta_{i} \hat{l}_{i} \cdot \hat{s}_{i}$
Table 1. Calculated atomic transitions and errors from the sodium ground state, as compared to multiplet-averaged experimental data (Moore 1971) (all in $\mathrm{cm}^{-1}$ ).

\begin{tabular}{lccc}
\hline \hline Level & Present & $\operatorname{Exp}$ & $\Delta$ \\
\hline $3 \mathrm{p}$ & 16944 & 16967 & -23 \\
$4 \mathrm{~s}$ & 25728 & 25740 & -12 \\
$3 \mathrm{~d}$ & 29159 & 29172 & -13 \\
$4 \mathrm{p}$ & 30250 & 30271 & -21 \\
$5 \mathrm{~s}$ & 33195 & 33200 & -5 \\
\hline
\end{tabular}

The total Hamiltonian $H_{\mathrm{el}}+H_{\mathrm{SO}}$ is expressed in the basis set of the eigenstates (here with $M_{s}= \pm \frac{1}{2}$ ) of the purely electrostatic Hamiltonian $H_{\mathrm{el}}$. The spin-orbit coupling between the molecular many-electron doublet states $\Phi_{k \sigma}$, approximated at this step as single determinants with the same closed shell $\sigma_{g}^{2} \mathrm{H}_{2}$ subpart, is isomorphic to that between the singly occupied molecular spinorbitals $\phi_{k \sigma}$, correlated with the six $p$ spin-orbitals of the alkali atom ( $k$ labels the space part and $\sigma=\alpha, \beta$ labels the spin projection).

The Cohen and Schneider approximation consists in assigning these matrix elements to their asymptotic atomic values,

$\left\langle\Phi_{k \sigma}\left|H_{\mathrm{SO}}\right| \Phi_{l \tau}\right\rangle=\left\langle\phi_{k \sigma}(\infty)\left|h_{\mathrm{SO}}\right| \phi_{l \tau}(\infty)\right\rangle$.

The scheme makes no a priori assumption about the magnitude of spin-orbit coupling versus pure electrostatic interactions and allows general intermediate coupling. The main question for the applicability of the scheme in a basis of adiabatic states is the transferability of the atomic SO integrals to the molecular case, because of configurational mixing. Such a situation characterizes the short-distance interaction between the repulsive state correlated with the $3 p$ configuration (either $2^{2} \Sigma^{+}$or $2^{2} \mathrm{~A}_{1}$, depending on the symmetry) and the attractive 4 s state $\left(3^{2} \Sigma^{+}\right.$or $\left.3^{2} \mathrm{~A}_{1}\right)$. This means that the atomic spin-orbit coupling is not transferable at short distance in the adiabatic basis. Although this is not essential since the gap with the ${ }^{2} \Pi$ components becomes large at short distance, we have taken the variation of the coupling scheme of the $3 \mathrm{p}, 4 \mathrm{~s}$ states into account in the following way. First, we achieved a diabatization of the $3 \mathrm{p} / 4 \mathrm{~s}$ anticrossing states in the ${ }^{2} \Sigma^{+}\left(\operatorname{resp}^{2} \mathrm{~A}_{1}\right)$ manifold, defining quasi-diabatic states $\tilde{\Phi}_{k}(k=3 \mathrm{p}, 4 \mathrm{~s}$ omitting the spin mention for convenience at this stage) as states with a constant transition dipole moment from the ground state. The adiabatic states are related to the latter through a $2 \times 2$ unitary transform at each distance $R$ depending on a mixing angle $\theta$. For the colinear case, the diabatic-to-adiabatic transformation is defined as

$\Phi\left(2^{2} \Sigma^{+}\right)=\cos \theta \tilde{\Phi}_{3 \mathrm{p}}\left({ }^{2} \Sigma^{+}\right)+\sin \theta \tilde{\Phi}_{4 \mathrm{~s}}\left({ }^{2} \Sigma^{+}\right)$

$\Phi\left(3^{2} \Sigma^{+}\right)=-\sin \theta \tilde{\Phi}_{3 \mathrm{p}}\left({ }^{2} \Sigma^{+}\right)+\cos \theta \tilde{\Phi}_{4 \mathrm{~s}}\left({ }^{2} \Sigma^{+}\right)$,

and the same transformation holds in the $C_{2 v}$ symmetry for ${ }^{2} \mathrm{~A}_{1}$ states.

Assuming the conservation of the transition dipole moments from the ground state to the quasi-diabatic states along the internuclear distance, the transition moments to the adiabatic states can be related to the former ones. For instance, the transition moment from the ground state $\Phi_{0}$ to the MRCI spin-orbit-less eigenstate $\Phi_{3 p}$ is

$\mu(R)=\left\langle\Phi_{0}|z| \Phi_{3 \mathrm{p}}\right\rangle=\left\langle\Phi_{0}|z| \cos \theta \tilde{\Phi}_{3 \mathrm{p}}\right\rangle=\cos \theta \times \mu_{3 \mathrm{p}}^{a t}$,

where $\mu(R)$ is the MRCI spin-orbit-less molecular adiabatic transition moment from the ground state to eigenstate $\Phi_{3 \mathrm{p}}$, and $\mu^{a t}$ 

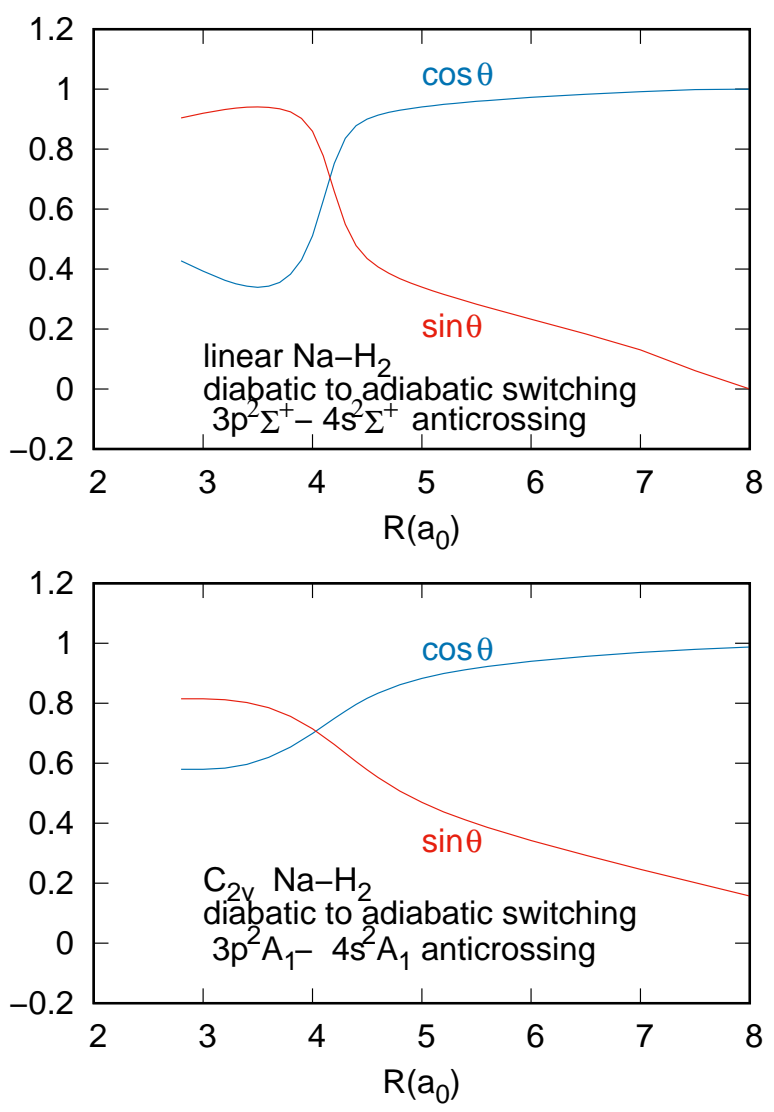

Fig. 4. Coefficients $(\cos \theta$ and $\sin \theta)$ of the $3 \mathrm{p} / 4 \mathrm{p}$ diabatization in $C_{\infty v v}$ (top) and $C_{2 v}$ symmetries (bottom).

is its atomic or asymptotic value $\left(2.537 a_{0}^{3}\right)$. Such a transformation was only carried for distances at which the molecular dipole moment is less than its atomic value. In the medium range around $R=10-11 a_{0}$, the adiabatic dipole transition moment reaches a very shallow maximum above its asymptotic value, that is $2.565 a_{0}^{3}$ for $C_{\infty v}$ and $2.549 a_{0}^{3}$ for $C_{2 v}$. We note that complementarily, the $\Phi_{4 \mathrm{~s}}$ state, asymtotically characterized by a vanishing transition dipole moment, acquires a non-zero coupling with the ground state with a $\sin \theta$ dependency. The evolution of the mixing along the internuclear distance is shown in Fig. 4. We obtain a $8 \times 8$ spin-orbit coupling matrix for the $3 \mathrm{p} / 4 \mathrm{~s}$ states, which is given in the Appendix.

The spin-orbit energy splitting ${ }^{2} P_{\frac{3}{2}}-{ }^{2} P_{\frac{1}{2}}$ of the $3 p$ levels of sodium is $17.19 \mathrm{~cm}^{-1}\left(=\frac{3}{2} \zeta\right)$ (Moore 1971). No such diabatization was considered for the $4 \mathrm{p}$ configuration which has been treated via a $6 \times 6$ coupling matrix $($ e.g $\cos \theta=1)$ and a $\zeta$ constant equal to two third of the $5.58 \mathrm{~cm}^{-1}$ experimental splitting of the $4 p$ manifold (Moore 1971). Spin-orbit coupling for the $3 \mathrm{~d}$ configuration has been neglected. The diagonalization of the total $H_{\mathrm{el}}+H_{\mathrm{SO}}$ matrix at each internuclear distance provides the final energies $E_{\mathrm{m}}^{\mathrm{SO}}$ and eigenstates $\Psi_{\mathrm{m}}^{\mathrm{SO}}$.

The transition dipole moments shown in Fig. 5 between the spin-orbit states were determined by recombining the adiabatic MRCI dipole moments over the coefficients $c_{k \sigma}^{n}$ of the spin-orbit states $\Psi_{\mathrm{m}}^{\mathrm{SO}}$,

$\mathbf{D}_{m n}^{\mathrm{SO}}=\left\langle\Psi_{m}^{\mathrm{SO}}|\mathbf{D}| \Psi_{n}^{\mathrm{SO}}\right\rangle=\sum_{k \sigma, l \tau} c_{k \sigma}^{m} c_{l \tau}^{n}\left\langle\Phi_{k \sigma}|\mathbf{D}| \Phi_{l \tau}\right\rangle \delta_{\sigma \tau}$.

In the following section, we only focus on the states correlated with the $3 \mathrm{p}$ manifold which determine the $\mathrm{Na}$ resonance

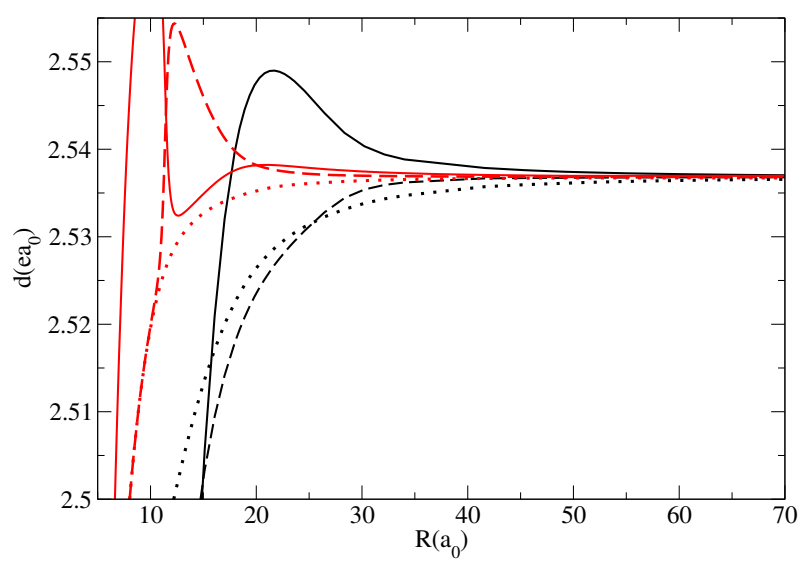

Fig. 5. Transition dipole moment for the $B-X$ (full line), $A^{2} \mathrm{P}_{3 / 2}-X$ (dotted line), and $A^{2} \mathrm{P}_{1 / 2}-X$ (dashed line) transitions of the $\mathrm{Na}-\mathrm{H}_{2}$ molecule for the $C_{\infty \infty v}$ (red curves) and $C_{2 v}$ symmetries (black curves); $B-X$ (full line), $A^{2} \mathrm{P}_{3 / 2}-X$ (dotted line), and $A{ }^{2} \mathrm{P}_{1 / 2}-X$ (dashed line).

lines. We evaluate the line parameters and collisional profiles for relevant temperatures and densities that are appropriate for modeling brown dwarf stars and hot-Jupiter-mass planets.

\section{Temperature and density dependence of the $\mathrm{Na}$ resonance lines}

In Allard et al. (1999), we derived a classical path expression for a pressure-broadened atomic spectral line shape that allows for an electric dipole moment that is dependent on the position of perturbers. This treatment has improved the comparison of synthetic spectra of brown dwarfs with observations (Allard et al. $2003,2007 b$ ). This approach to calculating the spectral line profile requires knowledge of molecular potentials with high accuracy because the shape and strength of the line profile are very sensitive to the details of the molecular potential curves describing the $\mathrm{Na}-\mathrm{H}_{2}$ collisions. Sodium is the most abundant alkali in cool dwarf atmospheres and is mostly responsible for the shape of the optical spectrum. With precise potentials and a complete line shape theory, major improvements in the theoretical description of pressure broadening have been made compared to the commonly used van der Waals broadening in the impact approximation (Burrows \& Volobuyev 2003; Allard et al. 2003, 2012a,b).

\subsection{Study of the line parameters}

The impact theories of pressure broadening (Baranger 1958; Kolb \& Griem 1958) are based on the assumption of sudden collisions (impacts) between the radiator and perturbing atoms, and are valid when frequency displacements $\Delta \omega=\omega-\omega_{0}$ and gas densities are sufficiently small.

In impact broadening, the duration of the collision is assumed to be small compared to the interval between collisions, and the results describe the line within a few line widths of center. One outcome of our unified approach is that we may evaluate the difference between the impact limit and the general unified profile, and establish with certainty the region of validity of an assumed Lorentzian profile. In the planetary and brown dwarf upper atmospheres the $\mathrm{H}_{2}$ density is of the order of $10^{16} \mathrm{~cm}^{-3}$ in the region of line core formation.

The line parameters presented in Allard et al. (2007c, $2012 a, b)$ were obtained using the pseudo-potentials of 


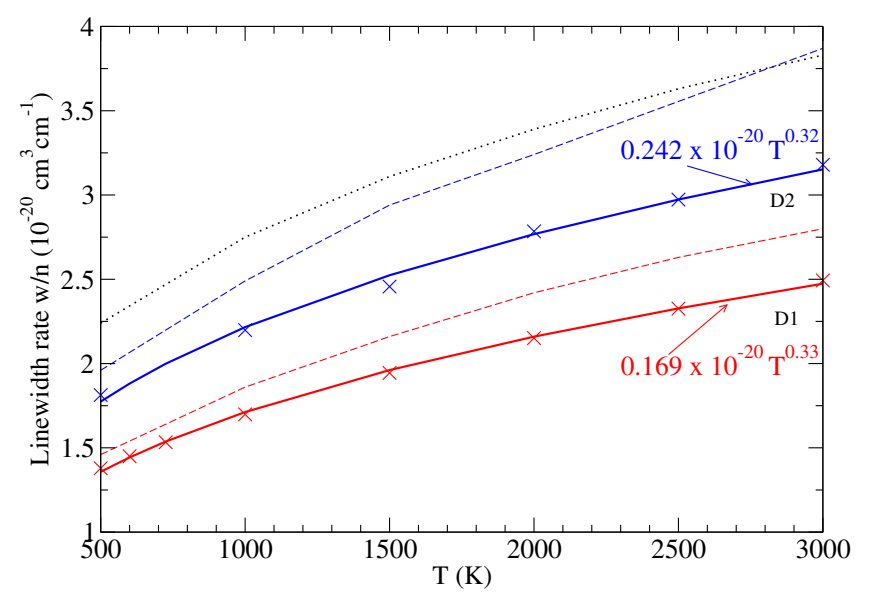

Fig. 6. Variation with temperature of the half-width of the $D 2$ (blue curves) and $D 1$ (red curves) lines of $\mathrm{NaI}$ perturbed by $\mathrm{H}_{2}$ collisions. New ab initio potentials (full line), pseudo-potentials of Rossi \& Pascale (1985) (dashed lines), and the van der Waals potential (black dotted lines).

Rossi \& Pascale (1985). To predict the impact parameters the intermediate- and long-range part of the potential energies need to be accurately determined. While the ab initio potentials presented in Allard et al. (2012a) allowed a better determination of the line wing, they were not accurate enough to determine the line parameters. With the improved potentials the half width at half-maximum $w$ is linearly dependent on $\mathrm{H}_{2}$ density, and a power law in temperature is given for the D1 line by

$w=0.169 \times 10^{-20} n_{\mathrm{H} 2} T^{0.33}$,

and for the $\mathrm{D} 2$ line is given by

$w=0.242 \times 10^{-20} n_{\mathrm{H} 2} T^{0.39}$.

where $w$ is in $\mathrm{cm}^{-1}, n_{\mathrm{H} 2}$ in $\mathrm{cm}^{-3}$, and $T$ in $\mathrm{K}$. These expressions accurately represent the numerical results as shown in Fig. 6, and may be used to compute the widths for temperatures of stellar or planetary atmospheres from 500 up to at least $3000 \mathrm{~K}$.

\subsection{Line satellite}

Since the first $\mathrm{Na}-\mathrm{H}_{2}$ pseudo-potentials were obtained by Rossi \& Pascale (1985), significant progress in the description of $\mathrm{NaH}_{2}$ potentials has been achieved by Burrows \& Volobuyev (2003), Santra \& Kirby (2005) and Allard et al. (2012a). Blue satellite bands in alkali- $\mathrm{He} / \mathrm{H}_{2}$ profiles can be predicted from the maxima in the difference potentials $\Delta V$ for the $B-X$ transition. Figures 1 and 2 of Allard et al. (2012a) present the ab initio potential curves without spin-orbit coupling for the $3 s$ and $3 p$ of S11 compared to pseudo-potentials of RP85. It is seen there that the major difference with respect to S11 is that RP85 potentials are systematically less repulsive. This difference affects the blue satellite position. The $\mathrm{NaH}_{2}$ line satellite is closer to the main line than obtained with RP85 (Fig. 5 of Allard et al. 2012a). We observe this effect on synthetic spectra in the following section. On the red side, the $\mathrm{NaH}_{2}$ wings match the profiles from the RP85 potentials.

Figures 7-9 show the sensitivity of the line wings to pressure and temperature. The density effect on the shape of the blue wing is highly significant when the $\mathrm{H}_{2}$ density becomes larger than $10^{20} \mathrm{~cm}^{-3}$. We notice a first line satellite at $5170 \AA$ in Fig. 7. A second satellite due to multiple-perturber effects appears as

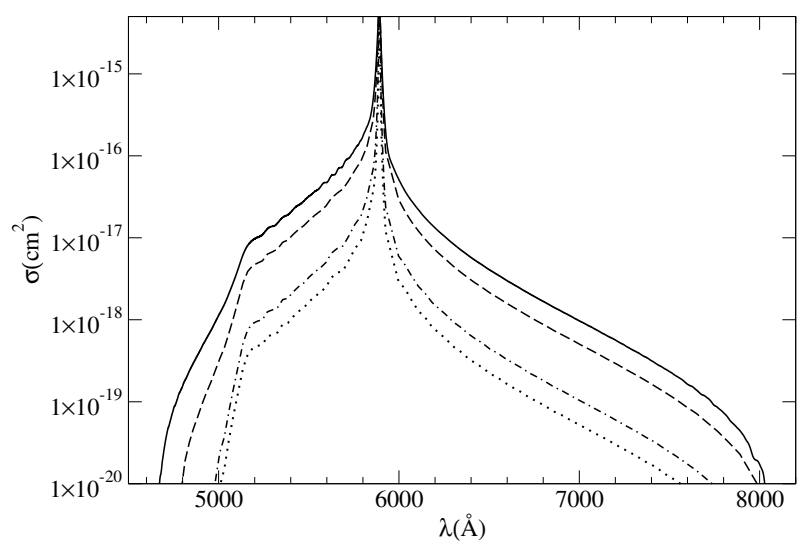

Fig. 7. Variation with the density of $\mathrm{H}_{2}$ of the $D 2$ component (from top to bottom $n_{\mathrm{H} 2}=10^{21}, 5 \times 10^{20}, 10^{20}$ and $5 \times 10^{19} \mathrm{~cm}^{-3}$ ). The temperature is $1500 \mathrm{~K}$.

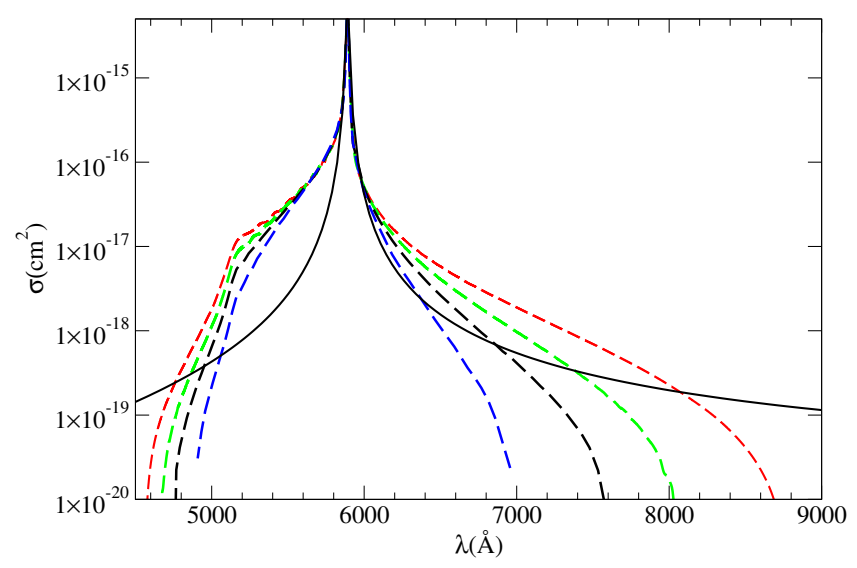

Fig. 8. Variation of the absorption cross sections of the 3s-3p D2 line component with temperature (from top to bottom $T=2500,1500$, 1000 , and $600 \mathrm{~K})$ for $n_{\mathrm{H} 2}=10^{21} \mathrm{~cm}^{-3}$. The Lorentzian profile for $1000 \mathrm{~K}$ is overplotted (black full line).

a shoulder at about $4800 \AA$ for $n_{\mathrm{H} 2}=10^{21} \mathrm{~cm}^{-3}$. The density dependence of the far blue wing arises from multiple-perturber effects and is not linear in density. Figures 8 and 9 show the absorption cross section of the resonance line of $\mathrm{Na}$ compared to the Lorentzian profiles calculated using the line widths presented in Fig. 6, for $T=1000 \mathrm{~K}$. The blue line wings shown in Fig. 8 are almost unchanged with increasing temperature whereas the red wings extend very far as temperature increases.

\subsection{Opacity tables}

For the implementation of alkali lines perturbed by helium and molecular hydrogen in atmosphere codes, the line opacity is calculated by splitting the profile into a core component described with a Lorentzian profile, and the line wings computed using an expansion of the autocorrelation function in powers of density. Here we briefly review the use of a density expansion in the opacity tables.

The spectrum $I(\Delta \omega)$ can be written as the Fourier transform (FT) of the dipole autocorrelation function $\Phi(\mathrm{s})$ (Allard et al. 1999),

$I(\Delta \omega)=\frac{1}{\pi} \operatorname{Re} \int_{0}^{+\infty} \Phi(s) e^{-i \Delta \omega s} \mathrm{~d} s$, 


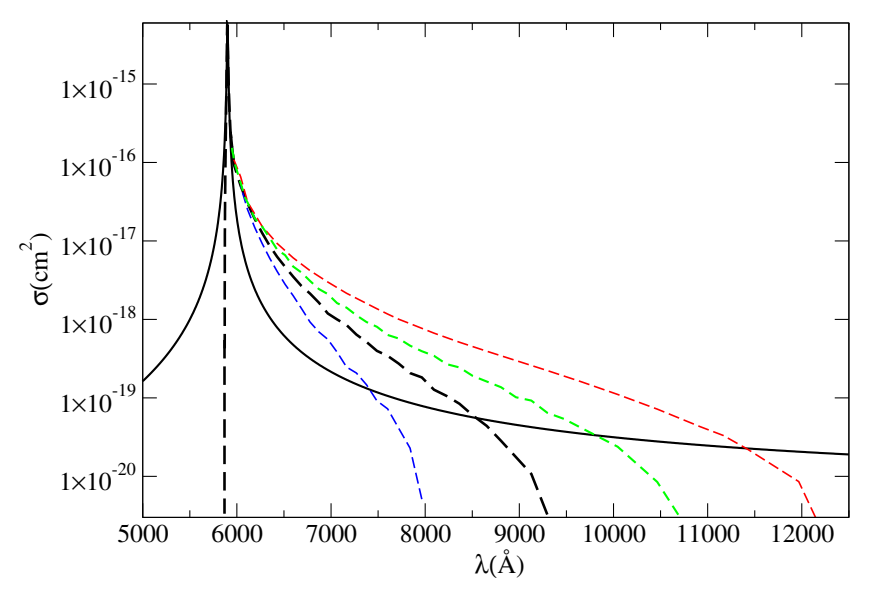

Fig. 9. Variation of the absorption cross sections of the 3s-3p D1 line component with temperature (from top to bottom $T=2500,1500$, 1000 , and $600 \mathrm{~K}$ ) for $n_{\mathrm{H} 2}=10^{21} \mathrm{~cm}^{-3}$. The Lorentzian profile for $1000 \mathrm{~K}$ is overplotted (black full line).

where $\Delta \omega$ is the angular frequency difference from the unperturbed center of the spectral line. The autocorrelation function $\Phi(s)$ is calculated with the assumptions that the radiator is stationary in space, the perturbers are mutually independent, and in the adiabatic approach the interaction potentials give contributions that are scalar additive. This last simplifying assumption allows us to calculate the total profile $I(\Delta \omega)$ when all the perturbers interact, as the FT of the $N$ th power of the autocorrelation function $\phi(s)$ of a unique atom-perturber pair. Therefore,

$\Phi(s)=(\phi(s))^{N}$,

that is to say, we neglect the interperturber correlations. We obtain for a perturber density $n_{\mathrm{p}}$

$\Phi(s)=e^{-n_{\mathrm{p}} g(s)}$,

where decay of the autocorrelation function with time leads to atomic line broadening. When $n_{\mathrm{p}}$ is high, the spectrum is evaluated by computing the FT of Eq. (11). The real part of $n_{p} g(s)$ damps $\Phi(s)$ for large $s$ but this calculation is not feasible when extended wings have to be computed at low density because of the very slow decrease of the autocorrelation function. An alternative is to use the expansion of the spectrum $I(\Delta \omega)$ in powers of the density described in Royer (1971).

We split the exponent $g(s)$ in Eq. (11) into a "locally averaged part" $g_{\text {av }}(s)$ and an "oscillating part" $g_{\mathrm{osc}}(s)$ by convolving $g(s)$ with a Gaussian $A(s)$ :

$g_{\mathrm{av}}(s)=A(s) * g(s)$,

and

$g_{\mathrm{osc}}(s)=g(s)-g_{\mathrm{av}}(s)$,

where the asterisk stands for a convolution product.

We can write

$g(s)=g_{\mathrm{av}}(s)+g_{\mathrm{osc}}(s)$.

At large values of $s, g(s)$ becomes linear in $s, g_{\text {osc }}$ vanishes, and the oscillating part remains bounded which allows us to expand $e^{-n_{\mathrm{p}} g_{\mathrm{osc}}(s)}$ in powers of $n_{\mathrm{p}} g_{\mathrm{osc}}(s)$; Eq. (11) becomes

$\Phi(s)=e^{-n_{\mathrm{p}} g_{\mathrm{av}}(s)}\left[1-n_{\mathrm{p}} g_{\mathrm{osc}}(s)+\frac{n_{\mathrm{p}}^{2}}{2 !}\left[g_{\mathrm{osc}}(s)\right]^{2}+\ldots\right]$.
The complete profile is given by the FT of Eq. (14):

$I(\Delta \omega)=I_{c}(\Delta \omega) *\left[\delta(\Delta \omega)-n_{p} I_{w}(\Delta \omega)+\frac{n_{p}^{2}}{2 !}\left[I_{w}(\Delta \omega)\right]^{* 2}+\ldots\right]$,

where $I_{c}(\Delta \omega)=\mathrm{FT}\left[e^{-n_{\mathrm{p}} g_{\mathrm{av}}(s)}\right]$ forms the core of the line profile and $I_{w}(\Delta \omega)=\mathrm{FT}\left[g_{\text {osc }}(s)\right]$ is responsible for the wing.

This method gives the same results as the FT of the general autocorrelation function (Eq. (11) without density expansion) at higher densities and has the advantage of including multiperturber effects at very low density when the general calculation is not feasible (see, e.g., Allard \& Alekseev 2014). The impact approximation determines the asymptotic behavior of the unified line shape correlation function. In this way the results described here are applicable to a more general line profile and opacity evaluation for the same perturbers at any given layer in the photosphere or planetary atmosphere.

When the expansion is stopped at the first order it is equivalent to the one-perturber approximation. Previous opacity tables were constructed to third order allowing us to obtain line profiles up to $N_{\mathrm{H} 2}=10^{19} \mathrm{~cm}^{-3}$. The new tables are constructed to a higher order allowing line profiles to $N_{\mathrm{H} 2}=10^{21} \mathrm{~cm}^{-3}$.

For a more direct comparison of the contributions of the two fine-structure components of the doublet it is convenient to use a cross-section $\sigma$ associated to each component. The relationship between the computed cross-section and the normalized absorption coefficient given in Eq. (9) is

$I(\Delta \omega)=\sigma(\Delta \omega) / \pi r_{0} f$,

where $r_{0}$ is the classical radius of the electron, and $f$ is the oscillator strength of the transition.

\section{Astrophysical applications}

\subsection{Self-luminous atmosphere}

In Fig. 10 we show the emission spectra for a cloud-free, selfluminous object (exoplanet or brown dwarf) at solar composition and varying effective temperature, calculated with petitCODE (Mollière et al. 2015, 2017). The atmospheric surface gravity was set to $\log (g)=4.5$, with $g$ in units of $\mathrm{cm} \mathrm{s}^{-2}$. The effective temperature was set to $T_{\text {eff }}=800,1500$, and $2500 \mathrm{~K}$, respectively. We calculated atmospheric structures and spectra using the old and new $\mathrm{Na}-\mathrm{H}_{2}$ line profiles. The opacity of $\mathrm{TiO}, \mathrm{VO}$, and $\mathrm{FeH}$ was neglected to make the alkali lines visible also for the highest-temperature model. The difference in Na blue wing absorption is clearly visible. We also show the self-consistent temperature profiles of the atmospheres for these cases, as calculated with petitCODE. In the example shown here the effect of the change in opacity of the Na wings on the temperature profile is too small to be seen and the solid and dashed lines overlap.

\subsection{Hot Jupiter}

In Fig. 11 we show the transmission spectra for cloud-free hotJupiter exoplanets at solar composition for a planetary effective temperature of $1800 \mathrm{~K}$, also calculated with petitCODE (Mollière et al. 2015, 2017). The planet mass and radius were chosen to be identical to the values of Jupiter, and an internal temperature of $T_{\text {int }}=200 \mathrm{~K}$ was used. The TiO/VO opacities were neglected. The host star was chosen to be a solar twin. We calculated atmospheric structures and spectra using the old and new $\mathrm{NaH}_{2}$ line profiles. The difference in Na blue wing absorption is clearly visible. We also show the self-consistent temperature profiles of the atmospheres for these cases, as calculated 


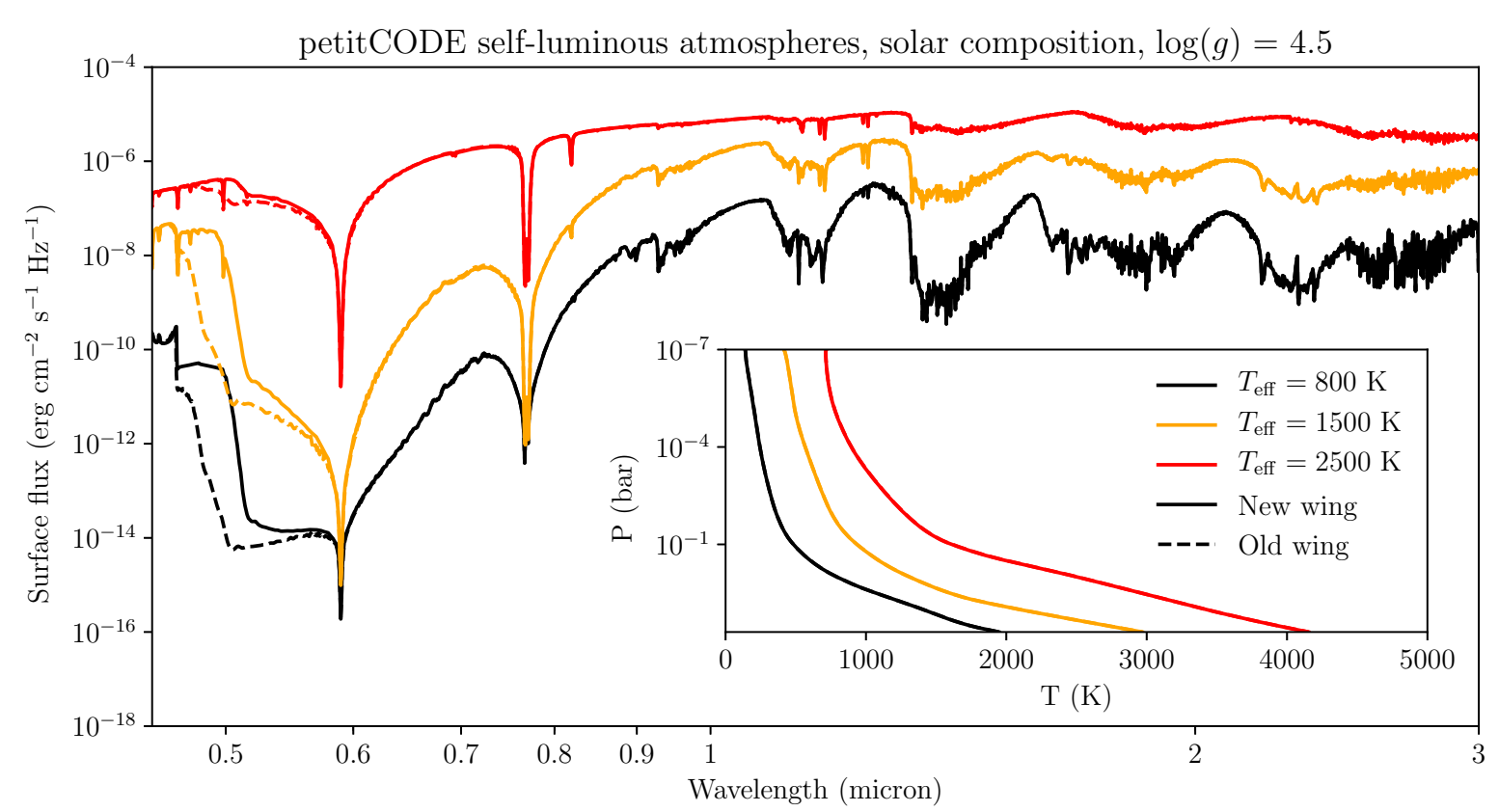

Fig. 10. Emission spectra for cloud-free, self-luminous objects (exoplanets or Brown Dwarfs) at solar composition and varying effective temperature, calculated with petitCODE (Mollière et al. 2015, 2017). The atmospheric surface gravity was set to $\log (g)=4.5$, with $g$ in units of $\mathrm{cm} \mathrm{s}^{-2}$. The black, orange, and red lines show the spectra for planets with $T_{\text {eff }}=800,1500$, and $2500 \mathrm{~K}$, respectively. Solid lines denote results obtained with the new Na wing profiles (presented in this paper), whereas dashed lines denote the results obtained with the Na lines reported in Allard et al. (2003). The inset plot shows the self-consistent temperature profiles for these cases, as calculated with petitCODE. In the example shown here the effect of the change in opacity of the $\mathrm{Na}$ wings is too small to be seen.

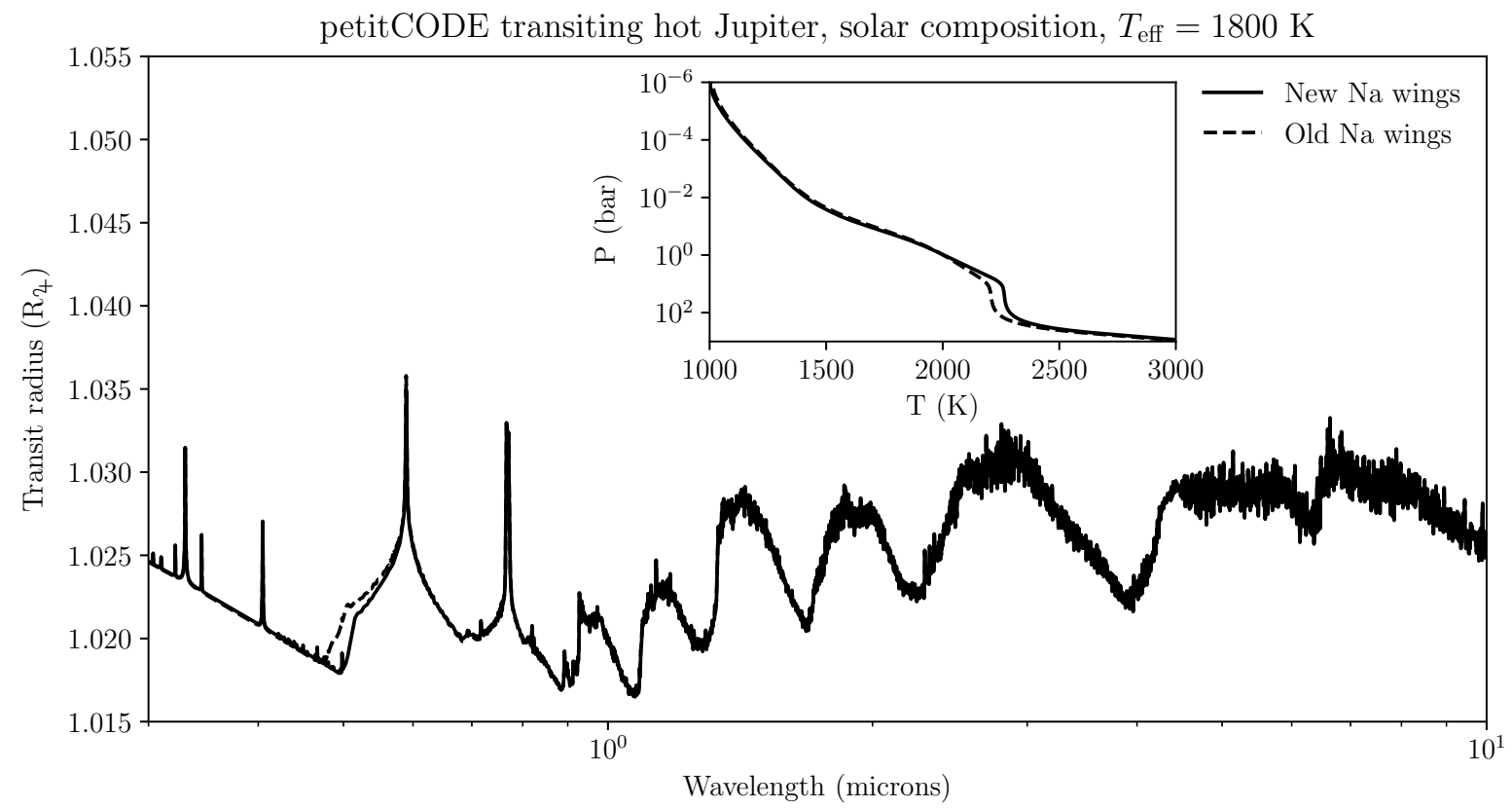

Fig. 11. Transmission radii for cloud-free, hot-Jupiter exoplanets at solar composition for a planetary effective temperature of $1800 \mathrm{~K}$, calculated with petitCODE (Mollière et al. 2015, 2017). The planet mass and radius were chosen to be identical to the values of Jupiter, and an internal temperature of $T_{\mathrm{int}}=200 \mathrm{~K}$ was used. The opacity of TiO and VO was neglected. The host star was chosen to be a solar twin. Solid lines denote results obtained with the new Na wing profiles (presented in this paper), whereas dashed lines denote the results obtained with the Na lines reported in Allard et al. (2003). The inset plot shows the self-consistent temperature profiles for these cases, as calculated with petitCODE. In the example shown here the weaker absorption in the new Na wing profiles leads to more greenhouse heating in the deep layers of the atmosphere, while the upper layers are slightly cooler than what was obtained with the old profiles.

with petitCODE. In the example shown here the weaker absorption in the new line profiles leads to more green house heating in the deep layers of the atmosphere, while the upper layers are slightly cooler than what was obtained with the old line profiles.

\section{Conclusion}

We performed theoretical calculations of the collisional profiles of the resonance lines of $\mathrm{Na}$ perturbed by $\mathrm{H}_{2}$ using a unified theory of spectral line broadening and high-quality ab initio 
potentials and transition moments. Figures 10 and 11 show that the perturbation of $\mathrm{Na}$ by $\mathrm{H}_{2}$ can be very important for the interpretation of visible spectra of brown dwarf and exoplanet atmospheres. We therefore suggest that the use of Lorentzian profiles is not appropriate for modeling the line wings, as Figs. 8 and 9 clearly show. Complete unified line profiles based on accurate atomic and molecular physics should be incorporated into analyses of exoplanet spectra when precise absorption coefficients are needed. Calculations are presented for the $D 1$ and $D 2$ lines from $T_{\text {eff }}=500 \mathrm{~K}-3000 \mathrm{~K}$ with a step size of $500 \mathrm{~K}$. Tables of the density expansion coefficients, an explanation of their use, and a program to produce line profiles to $N_{\mathrm{H} 2}=10^{21} \mathrm{~cm}^{-3}$ will be archived at the CDS.

\section{References}

Allard, N. F., \& Alekseev, V. A. 2014, Adv. Space Res., 54, 1248

Allard, N. F., Royer, A., Kielkopf, J. F., \& Feautrier, N. 1999, Phys. Rev. A, 60, 1021

Allard, N. F., Allard, F., Hauschildt, P. H., Kielkopf, J. F., \& Machin, L. 2003, A\&A, 411, L473

Allard, N. F., Spiegelman, F., \& Kielkopf, J. F. 2007a, A\&A, 465, 1085

Allard, F., Allard, N. F., Homeier, D., et al. 2007b, A\&A, 474, L21

Allard, N. F., Kielkopf, J. F., \& Allard, F. 2007c, EPJ D, 44, 507

Allard, N. F., Spiegelman, F., Kielkopf, J. F., Tinetti, G., \& Beaulieu, J. P. 2012a, A\&A, 543, A159

Allard, N. F., Kielkopf, J. F., Spiegelman, F., Tinetti, G., \& Beaulieu, J. P. 2012b, EAS Publ. Ser., 58, 239
Allard, N. F., Spiegelman, F., \& Kielkopf, J. F. 2016, A\&A, 589, A21 Arcangeli, J., Désert, J.-M., Line, M. R., et al. 2018, ApJ, 855, L30 Baranger, M. 1958, Phys. Rev., 111, 481

Baudino, J.-L., Mollière, P., Venot, O., et al. 2017, ApJ, 850, 150

Burrows, A., \& Volobuyev, M. 2003, ApJ, 583, 985

Burrows, A., Hubbard, W. B., Lunine, J. I., \& Liebert, J. 2001, Rev. Mod. Phys., 73, 719

Charbonneau, D., Brown, T. M., Noyes, R. W., \& Gilliland, R. L. 2002, ApJ, 568,377

Cohen, J. S., \& Schneider, B. 1974, J. Chem. Phys., 61, 3230

Kolb, A. C., \& Griem, H. 1958, Physical Review, 111, 514

Lothringer, J. D., \& Barman, T. S. 2019, ApJ, 876, 69

Lothringer, J. D., Barman, T., \& Koskinen, T. 2018, ApJ, 866, 27

Mollière, P., van Boekel, R., Dullemond, C., Henning, T., \& Mordasini, C. 2015, ApJ, 813, 47

Mollière, P., van Boekel, R., Bouwman, J., et al. 2017, A\&A, 600, A10

Moore, C. E. 1971, Bull. Am. Astron. Soc., 3, 154

Müller, W., Flesch, J., \& Meyer, W. 1984, J. Chem. Phys., 80, 3297

Nicklass, A., Dolg, M., Stoll, H., \& Preuss, H. 1995, J. Chem. Phys., 102, 8942

Nikolov, N., Sing, D. K., Fortney, J. J., et al. 2018, Nature, 557, 526

Pino, L., Ehrenreich, D., Wyttenbach, A., et al. 2018, A\&A, 612, A53

Rossi, F., \& Pascale, J. 1985, Phys. Rev. A, 32, 2657

Royer, A. 1971, Phys. Rev. A, 3, 2044

Santra, R., \& Kirby, K. 2005, J. Chem. Phys., 123, 214309

Sing, D. K., Fortney, J. J., Nikolov, N., et al. 2016, Nature, 529, 59

Snellen, I. A., Albretch, S., Mooij, E. J. W., \& Poole, R. S. L. 2008, A \& A, 487, 357

Tinetti, G., Vidal-Madjar, A., Liang, M.-C., et al. 2007, Nature, 448, 169

Werner, H.-J., Knowles, P. J., Knizia, G., Manby, F. R., \& Schütz, M. 2012, WIREs Comput. Mol. Sci., 2, 242

\section{Appendix A: SO matrix for $3 p$ and $4 s$ states}

$\left(\begin{array}{c}E_{3 p x} \\ 0 \\ i \frac{\zeta}{2} \\ 0 \\ 0 \\ \frac{\zeta}{2} \cos \theta \\ 0 \\ -\frac{\zeta}{2} \sin \theta\end{array}\right.$

0
$E_{3 p x}$
0
$-i \frac{\zeta}{2}$
$\frac{\zeta}{2} \cos \theta$
0
$-\frac{\zeta}{2} \sin \theta$
0

0
$i \frac{\zeta}{2}$
0
$E_{3 p y}$
$i \frac{\zeta}{2} \cos \theta$
0
$-i \frac{\zeta}{2} \sin \theta$
0

$\frac{\zeta}{2} \cos \theta$
0
$-i \frac{\zeta}{2} \cos \theta$
0
0
$E_{3 p z}$
0
0

$\left.\begin{array}{cc}0 & -\frac{\zeta}{2} \sin \theta \\ -\frac{\zeta}{2} \sin \theta & 0 \\ 0 & i \frac{\zeta}{2} \sin \theta \\ i \frac{\zeta}{2} \sin \theta & 0 \\ 0 & 0 \\ 0 & 0 \\ E_{4 s} & 0 \\ 0 & E_{4 s}\end{array}\right)$

$\left.\begin{array}{cc}0 & -\frac{\zeta}{2} \sin \theta \\ -\frac{\zeta}{2} \sin \theta & 0 \\ 0 & i \frac{\zeta}{2} \sin \theta \\ i \frac{\zeta}{2} \sin \theta & 0 \\ 0 & 0 \\ 0 & 0 \\ E_{4 s} & 0 \\ 0 & E_{4 s}\end{array}\right)$

